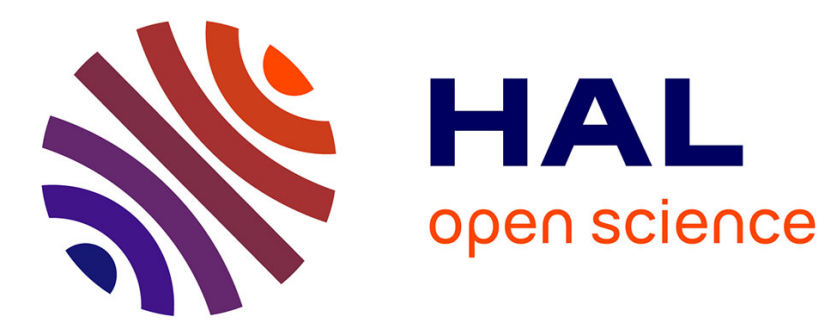

\title{
Underwater robot navigation around a sphere using electrolocation sense and Kalman filter
}

Vincent Lebastard, Christine Chevallereau, Ali Amrouche, Brahim Jawad, Alexis Girin, Frédéric Boyer, Pol-Bernard Gossiaux

\section{- To cite this version:}

Vincent Lebastard, Christine Chevallereau, Ali Amrouche, Brahim Jawad, Alexis Girin, et al.. Underwater robot navigation around a sphere using electrolocation sense and Kalman filter. Intelligent Robots and Systems (IROS), 2010 IEEE/RSJ International Conference on, Oct 2010, Taiwan. pp.4225-4230. hal-00704074

\section{HAL Id: hal-00704074 \\ https://hal.science/hal-00704074}

Submitted on 5 Jun 2012

HAL is a multi-disciplinary open access archive for the deposit and dissemination of scientific research documents, whether they are published or not. The documents may come from teaching and research institutions in France or abroad, or from public or private research centers.
L'archive ouverte pluridisciplinaire HAL, est destinée au dépôt et à la diffusion de documents scientifiques de niveau recherche, publiés ou non, émanant des établissements d'enseignement et de recherche français ou étrangers, des laboratoires publics ou privés. 


\title{
Underwater robot navigation around a sphere using electrolocation sense and Kalman filter
}

\author{
Vincent Lebastard, Christine Chevallereau, Ali Amrouche, Brahim Jawad, \\ Alexis Girin, Frederic Boyer, and Pol Bernard Gossiaux.
}

\begin{abstract}
The aim of this paper is to perform the navigation of an underwater robot equipped with a sensor using the electric sense. The robot navigates in an unbounded environment in presence of spheres. This sensor is inspired of some species of electric fish. A model of this sensor composed of $n$ spherical electrodes is established. The variations of the current due to the presence of the sphere is related to the model of Rasnow [3]. Unscented Kalman Filter is used to localize the robot with respect to the sphere and to estimate the size of the sphere. We show that bio-inspired motions improve the detection of the spheres. We illustrate the efficiency of the method in two cases: a two electrodes sensor and a four electrodes sensor.
\end{abstract}

\section{INTRODUCTION}

Lissmann and Machin [1] have demonstrated that weakly electric fishes use their electric organ discharges (EODs) to detect object with electric properties different from the surrounding medium. Near the fish an object with a higher conductivity than the medium brings locally more current on the skin whereas an object with less conductivity than the medium brings locally less current on the skin. The dependence is not only on the conductivity value but also on shape, dimension and distance from the skin. For a simple object like the sphere, B. Rasnow [3] gave the relation between the signal perturbation, i.e. the variation of the measured current, and the three parameters: the conductivity, the radius and the distance from the skin. The first problem in active electrolocation is to solve the ambiguity between these parameters. For instance, given a conductivity value, the same signal perturbation can come from both a bigger sphere that is situated far from the skin and from a smaller sphere that is close to the skin. Solberg and Al [4] have demonstrated the feasability of the detection of the sphere by the electric sense. Nevertheless they assume a value of conductivity and a value of the radius to perform the detection. Here we suppose we don't know the radius and the position of the sphere. Assuming a perfect conductive sphere or a perfect insulating sphere we perform the estimation of not only the position but also the radius of the sphere. This informations can be used to navigate with the electric sense. To realize this estimation we were inspired on some specific movements of the electric fish. Not only the electric sense but also the movement is important to perform the estimation. In

This work was supported by the European project ANGELS (ANGuilliform robot with ELectric Sense )

A. Amrouche is IRCCYN, Ecole des Mines de Nantes, Nantes, France. ali.amrouchedemn. fr

C. Chevallereau is IRCCYN, CNRS, Nantes, France. Christine. Chevallereaudirccyn.ec-nantes.fr fact Lannoo and $\mathrm{Al}$ [2] have reported the existence of specific movements for the electric fish in assessing a prey. Here we demonstrate the feasibility of the sphere recognition by the navigation and the electric sense. Our model of perception is bioinspired from the weakly electric fish. The sensor is composed of $n$ spherical electrodes of fixed potential values. Each object when it is polarized by the sensor creates a perturbation that is added to the initial potential values of the electrodes. We define the model of the sensor as the poly-spherical model. In the first part of this paper we present the poly-spherical model in a presence of a sphere. In the second part we present a method for the estimation of the parameters of the sphere. This method is based on the unscented Kalman filter. In the third part we illustrate the quality of the estimation with two examples: for a two electrodes sensor and for a four electrodes sensor. In each case we test the specific movements and we demonstrate the improvement of the estimation with these movements.

\section{ANALYTICAL MODEL}

As in the work of Rasnow [3], the model of electric sense is restricted to the resistive effects offered by the environments to the currents generated by the polarizations of the fish body. In these conditions, the principle of the electrolocation can be reproduced and modeled by considering the fish on the sensor copying it as a set of conducting annular electrodes aligned on a rigid slender body.

\section{A. The poly-spherical model in a presence of a sphere}

The geometry of the sensor allows one to approximate it with a good accuracy by a set of spherical electrodes aligned on the rigid body axis according to what we call a polyspherical model. We illustrate in the Fig. 1 such a model in the presence of a spherical object. The sensor is composed of $n$ spherical electrodes with an emitter and $n-1$ receivers at given potential values.

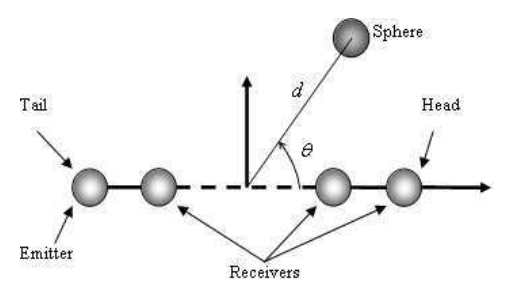

Fig. 1. The poly-spherical model in a presence of a sphere.

We derive our formalism from the equation of continuity: $\vec{\nabla} \cdot \vec{j}+\frac{\partial \rho}{\partial t}=0$ where $\vec{j}$ is the density current vector and $\rho$ is 
the volume charge density. The condition of stationarity leads to: $\vec{\nabla} \cdot \vec{j}=0$. The Ohm's law $\vec{j}=\gamma \vec{E}$ leads to $\gamma \vec{\nabla} \cdot \vec{E}+$ $\overrightarrow{\nabla \gamma} \cdot \vec{E}=0$ where $\vec{E}$ is the applied field. Considering that the conductivity of the domain in which the sensor is immersed is piece-wise constant (i.e. the fluid and the object are modeled as two Ohmic conducting homogeneous materials), we have in the fluid domain: $\vec{\nabla} \gamma=\overrightarrow{0}$, and consequently: $\vec{\nabla} \cdot \vec{E}=0$. Where we recognize the local form of the Gauss law in the case where all the electric charges are situated on the boundaries of the two Ohmic media (the electrodes and the sphere). Suppose now that on each electrode i we have the charge $q_{i}$ the potential of an electrode is simply deduced from the relation:

$$
V_{i}=\frac{1}{4 \pi \varepsilon} \sum_{j=1}^{n} \frac{q_{j}}{L_{i, j}}
$$

with the distance between the electrodes $i$ and the electrodes $j$ is given as $L_{i, j}=\delta_{i, j} R+\frac{j-1}{n-1} L, \delta_{i, j}$ the Kronecker delta which value is 1 for $i=j$ and 0 for $i \neq j$, R the radius of an electrode, $\mathrm{L}$ the length of the sensor and $\varepsilon$ the electric permittivity of the surrounding medium. We suppose implicitly that the electrodes are separated with the same distance. Now suppose that a sphere is appearing in the environment. The potential perturbation $\delta V$ due to this sphere is according to the Rasnow model [3] :

$$
\delta V=\chi a^{3} \frac{\vec{E} \cdot \vec{r}}{\|r\|^{3}}
$$

where $a$ is the radius of the sphere, $\gamma_{s}$ is the conductivity of the sphere, $\gamma$ is the conductivity of the medium, $\chi=\frac{\gamma_{s}-\gamma}{\gamma_{s}+2 \gamma}$ the contrast factor which value is 1 for a perfect conductive sphere and -0.5 for a perfect insulating sphere. We assume here that the applied field $\vec{E}$ is constant across the sphere. $\vec{r}$ is the position vector that comes from the center of the sphere to a point $\mathrm{M}$ where the perturbation is created. In the presence of the sphere one can write :

$$
V_{i}=\frac{1}{4 \pi \varepsilon} \sum_{j=1}^{n} \frac{q_{j}}{L_{i, j}}+\delta V_{i}
$$

where $\delta V_{i}$ is the perturbation due to the sphere created on the surface of the electrode i. The applied field $\vec{E}$ comes from the $n$ electrodes :

$$
\vec{E}=\sum_{j=1}^{n} \frac{q_{j} \vec{r}_{j}}{4 \pi \varepsilon\left\|r_{j}\right\|^{3}}
$$

where $\vec{r}_{j}$ is the position vector that comes from the center of the electrode $\mathrm{j}$ to the center of the sphere. Using now the integral form of the Gauss law: $\int_{\partial S_{i}} \vec{E} \cdot \overrightarrow{d s}=\frac{q_{i}}{\varepsilon}$

where $S_{i}$ is the surface of the electrode $\mathrm{i}$ and combining with the Ohm's law the integral form of the Gauss law becomes: $\int_{\partial S_{i}} \frac{\vec{j}_{i}}{\gamma} \overrightarrow{d s}=\frac{q_{i}}{\varepsilon}$. Assuming now that the electrodes are perfectly spherical and not too close to polarize themselves, the density current vector $\vec{j}_{i}$ becomes constant over the surface of an electrode i. Thus we can write: $\frac{I_{i}}{\gamma}=\frac{q_{i}}{\varepsilon}$ where $I_{i}$ is the total current entering an electrode i. With all these considerations one can link the potential of the electrodes to the current entering the electrodes:

$$
\mathbf{V}_{\mathbf{e}}=\frac{1}{4 \pi \gamma} \mathbf{R I}_{\mathbf{e}}
$$

where $\mathbf{R}$ is the matrix of resistivity (of dimensions $n \times n$ ), $\mathbf{I}_{\mathbf{e}}=\left[\mathbf{I}, I_{n}\right]^{T} 1^{1}$ with $\mathbf{I}$ is the current vector of receivers, $I_{n}$ the current of the emitter, $\mathbf{V}_{\mathbf{e}}=\left[\mathbf{V}, V_{n}\right]^{T}, \mathbf{V}$ is the potential vector of receivers and $V_{n}$ the potential of the emitter. We impose potential difference between emitter and receptors $\mathbf{U}$ $\left(U_{i}=V_{i}-V_{n}\right)$ and we express $\mathbf{U}$ in function of $\mathbf{V}_{\mathbf{e}}$ :

$$
\mathbf{U}=\mathbf{P V} \text {. }
$$

with $\mathbf{P}=\left[I_{(n-1)},-1_{(n-1,1)}\right]$, where $-1_{(n-1,1)}$ is a column of $n-1$ terms equal to -1 . To respect the current neutrality $I_{1}+\ldots I_{n}=0$, we express $\mathbf{I}_{\mathbf{e}}$ in function of $\mathbf{I}: \mathbf{I}_{\mathbf{e}}=\mathbf{P}^{T} \mathbf{I}$. We express $\mathbf{U}$ in function of $\mathbf{I}$ :

$$
\mathbf{U}=\frac{1}{4 \pi \gamma} \mathbf{P R P}^{T} \mathbf{I}
$$

The matrix $\mathbf{R}$ is composed by the contribution of each object $\mathbf{R}=\mathbf{A}+\mathbf{S}$ where $\mathbf{A}$ is the sensor self influence and $\mathbf{S}$ is the sphere influence.

The elements $A_{i, j}$ of the matrix $\mathbf{A}$ are calculated as:

$$
A_{i, j}=\frac{1}{\frac{|i-j|}{n-1} L+\delta_{i, j} R}
$$

and the elements $S_{i, j}$ of the matrix $\mathbf{S}$ are calculated as:

$$
S_{i, j}=-a^{3} \chi \frac{\xi_{i} \xi_{j}+\eta^{2}}{\left(\sqrt{\xi_{i}^{2}+\eta^{2}}\right)^{3}\left(\sqrt{\xi_{j}^{2}+\eta^{2}}\right)^{3}}
$$

where $i=1 . . n$, and $j=1 . . n$ and

$$
\left\{\begin{array}{l}
\xi_{l}=\left(-d \cos (\theta)+\frac{L}{2}-\frac{l-1}{n-1} L\right), \quad 1=\mathrm{i} \text { or } \mathrm{j} \\
\eta=d \sin (\theta)
\end{array}\right.
$$

where $d$ the distance between the center of the sensor and the center of the sphere, $\theta$ the angle between the axis of the sensor and the direction joining the center of the sensor and the center of the sphere (see Fig.1). Note that, these parameters are related to the body mobile frame and do not require to introduce any absolute extraneous frame. From (6), the current intensity vector $\mathbf{I}$ in the presence of the sphere can be expressed as:

$$
\mathbf{I}=4 \pi \gamma\left(\mathbf{P R P}^{T}\right)^{-1} \mathbf{U}
$$

In the next sections, The measured current $\mathbf{I}$ will be used to locate and recognize the sphere.

\section{B. The sensor's range}

In this section, the goal is to determine the distance at which the sensor detects the sphere in an unbounded environment. The Fig. 2 shows the two characteristics distances: the axial and the lateral range. We compare the range of two kinds of sensors, a dipole ( $n=2$ electrodes) and a quadripole

\footnotetext{
${ }^{1}$ Notation ${ }^{T}$ means transposition
} 


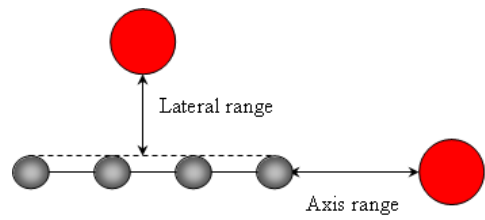

Fig. 2. Definition of axial and lateral range.

( $n=4$ electrodes). We evaluated the current measure noise in the test bench ${ }^{2}$ at $I_{b}=10^{-6} A$. We assume that the maximum range is obtained when the current $\|\mathbf{I}\|$ is in the same order than $I_{b}$. The length of the sensors is $L=0.2 \mathrm{~m}$ and the distance between two successive electrodes is $\frac{L}{n-1}$. Radius of the electrodes is $R=0.01 \mathrm{~m}$. The potential at the emitter is $2 \mathrm{~V}$ and at the receivers is $0 \mathrm{~V}$.

We place a sphere at different locations around the sensor in an $0.4 m \times 0.4 m$ area. For each position, we calculate the norm of the currents expressed us: $I_{s}=\left\|\mathbf{I}-\mathbf{I}_{\infty}\right\|$, where $\mathbf{I}_{\infty}$ (respectively I) is the current vector in an unbounded environment without the sphere (respectively with the sphere).

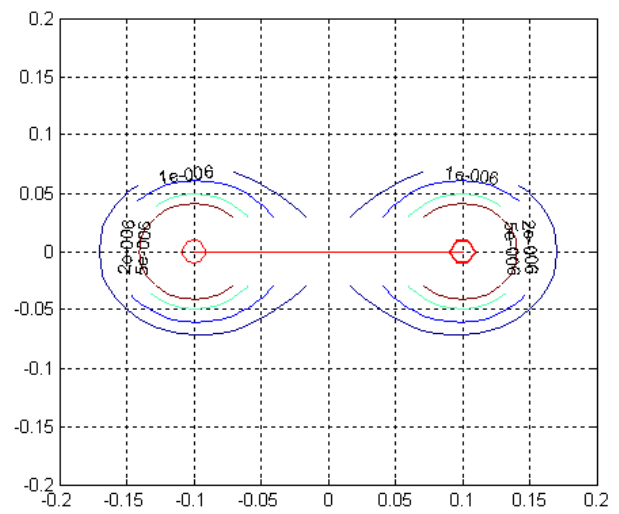

Fig. 3. The iso-current Is for the dipole.

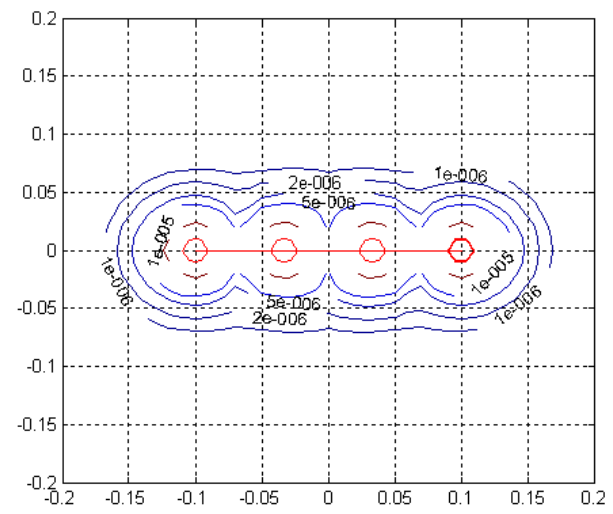

Fig. 4. The iso-current Is for the quadripole.

\footnotetext{
${ }^{2}$ Test bench can not be presented, we are waiting for patent.
}

In the Fig. 3 and Fig. 4, the curves display the current $I_{s}$ for aconductive sphere whit a radius $a=0.01 \mathrm{~m}$. In the Fig. 3, we observe clearly a "blind area" in the middle of the sensor. This characteristic exist if $L_{i, j}>>a$ i.e. bigger sphere can be "seen" by the dipole. We do not find this characteristic for the four electrodes sensor (see Fig. 4).

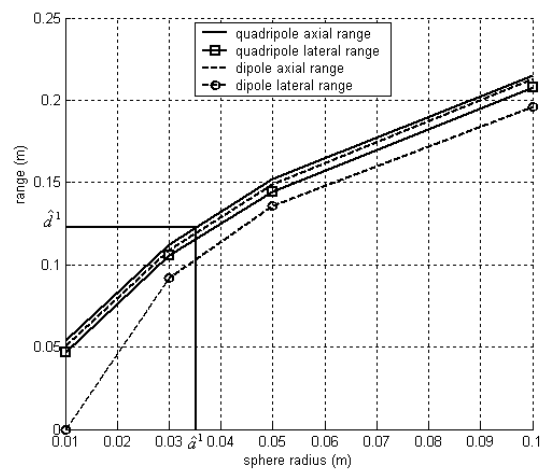

Fig. 5. The range of the sensor for several values of the sphere radius .

Fig. 5 shows the lateral and the axial range of the dipole and the quadripole for several values of a conductive sphere radius $a=[0.01,0.03,0.05,0.10] m$. The two sensors have similar axial range. The lateral and the axial ranges of the quadripole are also similar. A sphere of radius $a<0.05 \mathrm{~m}$ is better detected laterally by the quadripole than by the dipole. A sphere of radius $a \leq 0.01 \mathrm{~m}$ can not be detect laterally by the dipole.

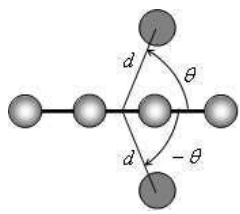

Fig. 6. The indistinguishable sphere positions.

The sensor is designed with symmetrical right-left shape. With this design it is impossible to distinguish the side where the object is [10] as shown in Fig. 6. Moreover, the dipole can not make the difference between all the positions of the sphere for the same iso-current (see Fig.3). To raise these problems, more informations is needed, we can obtain it by motions. We propose to use some specific bio-inspired motions.

\section{ELECTROLOCATION AND RECOGNITION OF THE SPHERE}

The calculation of the sphere parameters using the current mesure is an inverse problem. Inverse problems may be difficult or impossible to solve. For example, using the dipole we have only one measure witch is not enough to identify the sphere parameters, a solution is to use the observers. We propose to use the Unscented Kalman Filter to estimate the sphere parameters. The sphere parameters are:

- The sphere position: the distance $d$ and the angle $\theta$. 
- The sphere radius $a$.

- The sphere class: conductive or insulating, is given by contrast factor $\chi$.

\section{A. The motion model of the sensor}

In case of a real fish, the motion is ruled by its swimming dynamics. In our simplified case, the rigid sensor mimicking the fish is modeled as a single non-holonomic axle which motions parallel to the $y$ axis are forced to zero (see Fig.1). While being very simple, these kinematics reproduce the fact that a fish cannot swim along the lateral dimensions of its body. In these conditions, the sensor motion is simply parameterized by the linear velocity $V$ and the angular velocity $\omega$ (see Fig. 1). The sphere is assumed to be fixed and located with respect to the sensor furthermore sphere radius $a$ is constant. $V$ and $\omega$ define the input control vector $u=[V, \omega]^{T}$.

Based on Fig. 1, the motion model can be written:

$$
\left\{\begin{aligned}
\dot{d} & =-V \cos (\theta) \\
\dot{\theta} & =\omega+\frac{V \sin (\theta)}{d} \\
\dot{a} & =0
\end{aligned}\right.
$$

This model can also be written under a discrete first order form:

$$
\left\{\begin{array}{l}
d^{k+1}=d^{k}-V^{k} \cos \left(\theta^{k}\right) \Delta t \\
\theta^{k+1}=\theta^{k}+\left(\omega^{k}+\frac{V^{k} \sin \left(\theta^{k}\right)}{d^{k}}\right) \Delta t \\
a^{k+1}=a^{k}
\end{array}\right.
$$

Where $k$ denote the time step and $\Delta t$ is the sampling period. The state vector is $x^{k}=\left(d^{k}, \theta^{k}, a^{k}\right)^{T}$ and the control input vector is $u^{k}=\left(V^{k}, \omega^{k}\right)^{T}$. The evolution model 12 can be written as: $x^{k+1}=f\left(x^{k}, u^{k}\right)$ and the measure model (10) as: $y^{k}=I^{k}=g\left(x^{k}, \chi\right)$

\section{B. The observer}

One class of observer is based on the Kalman filter [5], [9], [6]. In case of nonlinear systems the Extended Kalman Filter (EKF) can be used. It requires to linearize the model of measures as well as that of motion. To avoid such a costly analytical linearization of the two models, the Unscented Kalman Filter (UKF) will be used [7]. In this case the linearization of the model is implicit and numerically defined via several "sigma points" i.e. based on Gaussian random variable. The correction and the definition of the sigmapoints are based on covariance matrix $P^{k}$. In the following, the discrete model of evolution (12) is used. The covariance matrix $P^{k}$ attached to the state variable $x^{k}$ defines the precision associated to the state. A small covariance implies that the variable is precisely known, a large covariance implies that the state is not precisely known. The evolution of the covariance matrix is defined by the UKF algorithm. Two main elements affect the behavior of the Kalman filter: the covariance matrix associated to the model of evolution $Q_{m}$ which defines if this model is well known or not and the covariance matrix of measure noise $Q_{s}$. The principle of the UKF is summarized in the following algorithm using recursive equations [9]:
- step k: the observed state is $\hat{x}^{k}$.

- $2 \mathrm{~N}+1$ sigma points are defined $\left(x \in \mathfrak{R}^{N}\right)$.

$$
\left\{\begin{array}{l}
\chi_{0}^{k}=\hat{x}^{k} \\
\chi_{i}^{k}=\hat{x}^{k}+\sqrt{(N+\kappa)\left(P^{k}+Q_{m}\right)} \\
\chi_{i+N}^{k}=\hat{x}^{k}-\sqrt{(N+\kappa)\left(P^{k}+Q_{m}\right)}
\end{array}\right.
$$

where $i=1, \ldots, N$ and $\kappa$ is the scale factor which can be chosen (in our case $\kappa=1$ ).

- The sigma points are weighted: $W_{0}=2 \kappa, W_{i}=1$ for $i=1, \ldots, N$

- State prediction: using the evolution model (12):

$$
\begin{aligned}
& \chi_{i, k+1 \mid k}=f\left(\chi_{i}^{k}, u^{k}\right), i=0, \ldots, 2 N \\
& \hat{x}_{k+1 \mid k}=\frac{1}{2(\kappa+N)} \sum_{i=0}^{2 N}\left(W_{i} \chi_{i, k+1 \mid k}\right) \\
& \Delta_{i, \chi, \hat{x}}=\chi_{i, k+1 \mid k}-\hat{x}_{k+1 \mid k}, i=0, \ldots, 2 N \\
& P_{k+1 \mid k}=\frac{1}{2(\kappa+N)} \sum_{i=0}^{2 N}\left(W_{i}\left[\Delta_{i, \chi, \hat{x}}\right]\left[\Delta_{i, \chi, \hat{x}}\right]^{T}\right)
\end{aligned}
$$

- Measure prediction: using the mesure model (10):

$$
\begin{aligned}
& y_{i, k+1 \mid k}=g\left(\chi_{i, k+1 \mid k}\right), i=0, \ldots, 2 N \\
& \hat{y}_{k+1 \mid k}=\frac{1}{2(\kappa+N)} \sum_{i=0}^{2 N}\left(W_{i} y_{i, k+1 \mid k}\right) \\
& \Delta_{i, y, \hat{y}}=y_{i, k+1 \mid k}-\hat{y}_{k+1 \mid k}, i=0, \ldots, 2 N \\
& P_{y y}=\frac{1}{2(\kappa+N)} \sum_{i=0}^{2 N}\left(W_{i}\left[\Delta_{i, y, \hat{y}}\right]\left[\Delta_{i, y, \hat{y}}\right]^{T}\right)+Q_{s} \\
& P_{x y}=\frac{1}{2(\kappa+N)} \sum_{i=0}^{2 N}\left(W_{i}\left[\Delta_{i, \chi, \hat{x}}\right]\left[\Delta_{i, y, \hat{y}}\right]^{T}\right)
\end{aligned}
$$

- Gain of the correction: $K^{k+1}=P_{x y} P_{y y}^{-1}$

- Correction: $\begin{aligned} & \hat{x}^{k+1}=\hat{x}_{k+1 \mid k}+K_{k+1}\left(y-\hat{y}_{k+1 \mid k}\right) \\ & P^{k+1}=P_{k+1 \mid k}-K_{k} P_{y y} K_{k}^{T}\end{aligned}$

$P^{k+1}=P_{k+1 \mid k}-K_{k} P_{y y} K_{k}^{T}$

\section{Electrolocation of A SPHERICAL OBJECT}

We assume that there is only one conducting sphere in the navigation environment of the sensor. The sphere radius is $5 \mathrm{~cm}$. If there are several spheres, we assume that every sphere is sufficiently far from the others so that the sensor will not detect two spheres at the same time.

\section{A. The observer initialization}

Before the sphere detection, the sensor moves in an unbounded environment. If the sensor detects a sphere, it will be at the limit of detection area at front of the sensor Fig. 7. The detection area is defined based on the range study Fig 4. At this instant, the observer starts and the contrast $\chi$ will be evaluated. If the current decreases, the sphere is declared to be insulating: $\chi=-0.5$. Conversely, if the current increases the sphere is conductive: $\chi=1$. We give an arbitrary initial value of the sphere radius $\hat{a}^{1} \in[0.01,0.1](\mathrm{m})$. The initial distance $\hat{d}^{1}$ is given based on the range study Fig.5. The system (11) is not observable [1] if the angle $\hat{\theta}$ is in the neighborhood of $0(\mathrm{rad})$. To avoid this singularity, the initial value of the angle $\theta^{1}=\frac{\pi}{4}$.

The initial covariance matrix $P^{1}$ is given according to sensor range study as: $P^{1}=\operatorname{diag}\left[0.1^{2}, 0.5^{2}, 0.05^{2}\right]$ The initial value of standard variation of the radius is 0.05 . According to 
Fig. 5, we chose 0.1 as the initial value of standard variation of the distance.

In the simulations of this study, the matrix $Q_{m}$ is chosen as following: $Q_{m}=\operatorname{diag}\left[0.01^{2}, 0.01^{2}, 0\right]$

The covariance matrix of measure noise $Q_{s}=I_{b}^{2} *$ $\mathbf{I}_{n-1 \times n-1}$, where $\mathbf{I}_{n-1 \times n-1}$ is the identity matrix, $n$ is the number of electrodes.

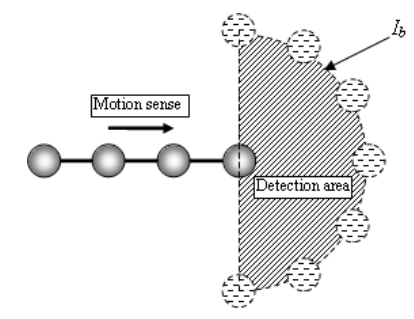

Fig. 7. Possible sphere positions at the detection instant.

\section{B. Simulations results and discussion}

The observer estimates the sphere parameters: the distance $\hat{d}$, the angle $\hat{\theta}$ and the sphere radius $\hat{a}$. The control law uses the estimated state. In the following example, the sphere is conductive and its real radius is $a=0.05 \mathrm{~m}$.

To recognize the sphere parameters, the sensor realize the following bio-inspired motions [2]:

- back and forth: The input control vector is $u^{k}=$ $\left[V^{k}, \omega^{k}\right]^{T}=\left[V_{0} \cos \left(\Omega_{V} k \Delta t\right), 0\right]^{T}$.

- back and forth with yawing: The input control vector is $u^{k}=\left[V^{k}, \omega^{k}\right]^{T}=\left[V_{0} \cos \left(\Omega_{V} k \Delta t\right), \omega_{0} \cos \left(\Omega_{\omega} k \Delta t\right)\right]^{T}$,

Where $\Omega$ is the pulsation, $V_{0}$ is the amplitude of the linear velocity and $\omega_{0}$ is the is the amplitude of the angular velocity.

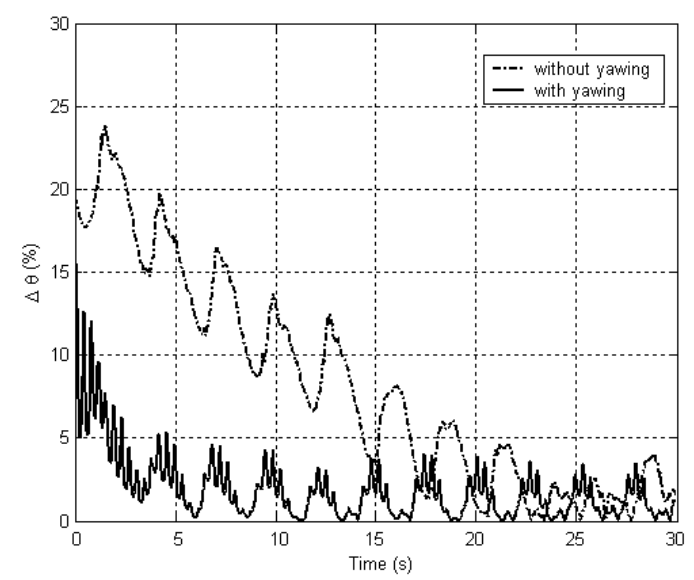

Fig. 8. Angle estimation errors using the bioinspired movement (B and B) with quadripole.

Fig. 8 shows the estimation error of the angle using the quadripole. For the two bio-inspired motions, the angle estimation error converge toward zero. The convergence is faster for the back and forth with yawing than without. In order to show the contribution of this bio-inspired motion

\begin{tabular}{|c|c|c|c|c|}
\hline \hline $\begin{array}{c}\text { The bio- } \\
\text { ispired } \\
\text { motion }\end{array}$ & $\begin{array}{c}\text { number } \\
\text { of elec- } \\
\text { trodes } \mathrm{n}\end{array}$ & $\begin{array}{c}\text { error } \\
\text { mean of } \\
d(\%)\end{array}$ & $\begin{array}{c}\text { error } \\
\text { mean of } \\
\theta(\%)\end{array}$ & $\begin{array}{c}\text { error } \\
\text { mean of } \\
a(\%)\end{array}$ \\
\hline $\mathrm{A}$ & $\mathrm{n}=2$ & 5.90 & 35.61 & 6.34 \\
& $\mathrm{n}=4$ & 3.30 & 12.56 & 6.07 \\
\hline $\mathrm{B}$ & $\mathrm{n}=2$ & 2.55 & 10.11 & 5.21 \\
& $\mathrm{n}=4$ & 1.70 & 8.75 & 3.47 \\
\hline $\mathrm{C}$ & $\mathrm{n}=2$ & 2.18 & 12.01 & 4.67 \\
& $\mathrm{n}=4$ & 1.24 & 3.29 & 2.08 \\
\hline $\mathrm{D}$ & $\mathrm{n}=2$ & 2.12 & 6.83 & 3.41 \\
& $\mathrm{n}=4$ & 1.02 & 0.82 & 2.01 \\
\hline \hline
\end{tabular}

TABLE I

SiMULATION RESULTS FOR THE MOTIONS

in the sphere recognition, we realize four tests described in Fig.9 using the two sensors: the dipole and the quadripole.

- Motion A: back and forth at front of the sphere.

- Motion B: back and forth at front of the sphere with yawing.

- Motion C: back and forth at side of the sphere.

- Motion D: back and forth at side of the sphere with yawing.

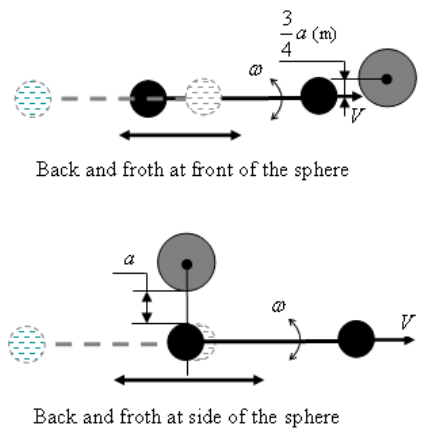

Fig. 9. Tested motions.

Table I lists the obtained results using the bio-inspired movements. The error mean is the mean error for all the duration of the simulation (here $k=1 . .1000$ ). With the motion (A), the observer can estimate the radius, the distance but the error mean of the angle is relatively important $(35.61 \%)$. With the motion (B), we observe a remarkable improvement of the observer estimation performances for the angle (the estimation error). The yawing moves away the angle $\theta$ from the singular value $(0 \mathrm{rad})$. When the sensor moves at side of the sphere (motions (C) and (D)), we obtain better results with yawing for quadripole. If we compare the results obtained for the motions (B) and (C) with the dipole, we observe that the estimation errors are closed. This two motions have the same effect on the angle $\theta$ moves away the angle from the singular value. The best estimations performance are obtained with the bio-inspired motion (D) using the quadrupole. 


\section{Electrolocation AND ORBITING AROUND THE SPHERE}

An example of navigation with the electric sens in presence of a sphere is given in Fig.10. We realize the following scenario:

- The quadripole moves in its environment until the detection of the sphere.

- Contrast evaluation $\chi$ and observer initialization (section IV-A).

- Specific motion back and forth with yawing and estimation of the sphere parameters $\hat{d}, \hat{\theta}$ and $\hat{a}$.

- When the observer converge, the quadripole orbiting around the sphere with a constance distance $R_{0}$ Fig. 10 .

In this simulation, the sphere is conductive and its real radius is $a=0.05(m)$.

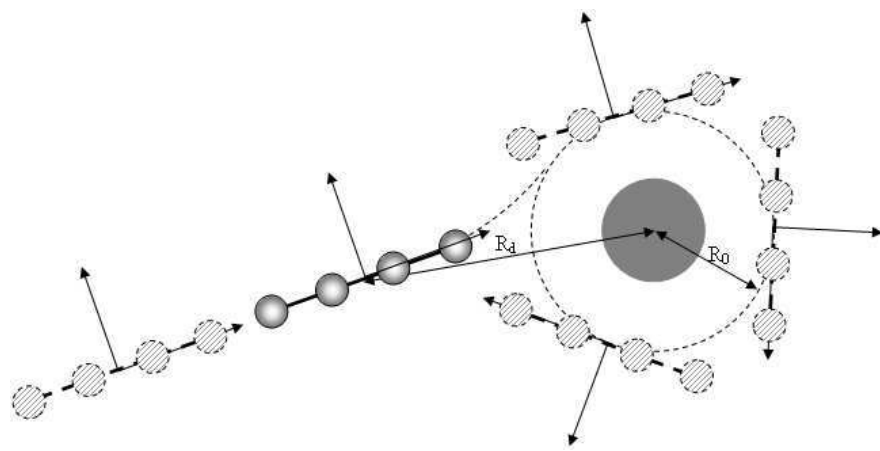

Fig. 10. Trajectory of electrolocation and orbiting around the sphere.

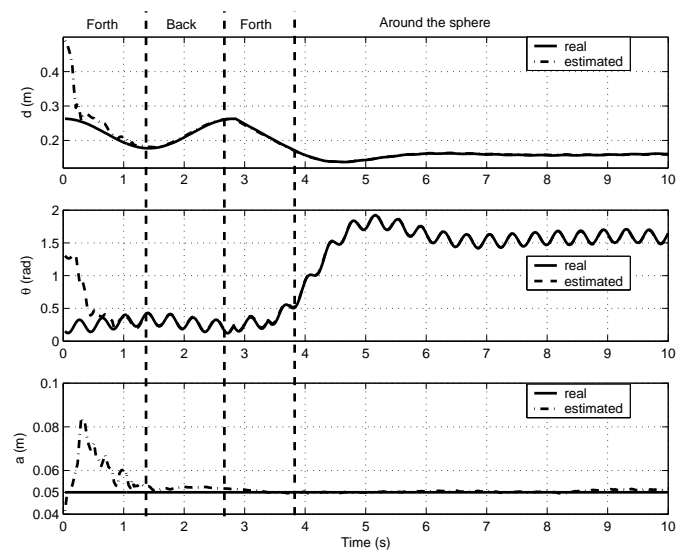

Fig. 11. Orbiting around the sphere using the quadripole with the motion (B).

Fig.11 shows the real and the estimated sphere parameters and Fig.12 shows the estimation errors of the sphere parameters. During the first forth, the standard deviation are over than the evolution model noise $Q_{m}$. So, the quadripole do again an other back and forth with yawing. At the end of this second forth, the estimation errors are less than the the evolution model noise $Q_{m}$. The sphere parameters are known, then the quadripole orbiting around the sphere.

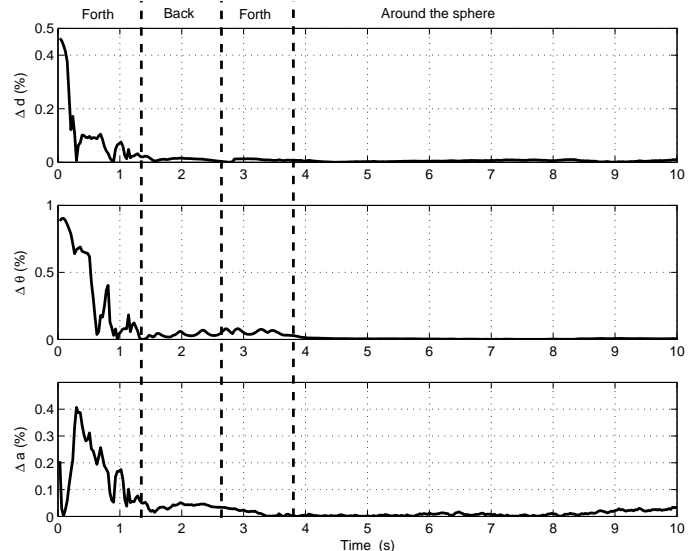

Fig. 12. Estimation errors.

\section{CONCLUSIONS}

In this paper, we propose bio-inspired motions to improve the localization and the recognition of sphere by the electric sense using the Unscented Kalman filter. Two sensors model are used: the dipole and the quadripole. Based on the range study, the quadripole is preferred than the dipole. According to the simulations results, the using of the bio-inspired motions improves the estimation of the sphere parameters. With the quadripole, the observer gives better results. This results give us the possibility to improve the underwater navigation with the electric sense by using the optimal combination between the sensor specifications and the bioinspired motions.

\section{ACKNOWLEDGMENTS}

The project ANGELS is funded by the European Commission, Information Society and Media, Future and Emerging Technologies (FET) contract number: 231845.

\section{REFERENCES}

[1] H. W. Lissman and K.E. Machin, The Mechanism of Object Location in Gymnarchus niloticus and similar fish, J.Exp.Biol. 35, 451-486, 1958.

[2] M. J. Lannoo and S. J. Lannoo, Why do Electric Fishes Swim Backwards?, Environmental biology of fishes, vol. 36, n2, pp. 157165 (1 p.), 1993.

[3] B. Rasnow. The Effect of sSimple Objects on the Electric Field of Apteronotus, Journal of Comparative Physiology A, vol. 36, 178(3): 397-411, 1996.

[4] J. R. Solberg, K.M Lynch and M. A. MacIver, Active Electrolocation for Underwater Target Localization, The International Journal of Robotics Research, Vol. 27, No.5, pp.529-548, 2008.

[5] M. A. MacIver and M. E. Nelson, Towards a Biorobotic Electrosensory System. Autonomous Robots, 11,263-266, 2001.

[6] R. Kalman, A New Approach to Linear Filtering and Prediction Problems , Transactions of the ASME - Journal of Basic Engineering , Vol. 82, pp.35-45, 1960

[7] H. Nijmeijer and A. J. Van der Schaft, Nonlinear Dynamical Control Systems. Springer-Verlag, 1990.

[8] S.G. Mohinder and P.A. Angus, Kalman Filtering Theory and Practice. Prentice hall, 1993.

[9] J. Julier Simon and K. Uhlmann Jeffrey, A New Extension of the Kalman Filter to Nonlinear Systems, Int. Symp. Aerospace/Defense Sensing, Simul. and Controls, pp.182-193, 1997.

[10] G. Baffet, F. Boyer and P. B. Gossiaux, Biomimetric Localization Using the electrolocation sense of The Electric Fish, International Conference on Robotics and Biomimetrics, Thailand, 2009. 